\title{
Le projet Accès-Cible de la Nuit des Musées lausannois: une expérimentation pour promouvoir l'accès des personnes handicapées à la culture
}

\section{Carine Bonsack, Monique Richoz, Lausanne}

\section{Un projet de la Nuit des Musées lausannois}

La Nuit des Musées lausannois (NDM) est un événement urbain qui, chaque année de 14 heures à quatre heures du matin, célèbre la culture en ouvrant les portes des musées. L'accent est mis sur une communication décalée et ludique qui se décline chaque année sur un thème différent. Il y a eu les animaux domestiques avec le collier de chien comme billet d'entrée et des slogans du type «Adoptez les musées et apprivoisez votre culture», la thématique de la mode avec des lunettes de soleil bling bling comme billet d'entrée et le slogan «Défilé Haute-Culture» ou celle de la crise avec le slogan «Sortez de la crise, entrez au musée!». A côté de sa programmation, chaque musée présente une animation pour petits et grands. Par ailleurs, l'événement concernant l'ensemble de la ville, les restaurants et les transports publics participent pleinement au projet. La manifestation se poursuit tard dans la nuit avec le «Musée éphémère», lieu de rencontre pour les adultes jusqu'à quatre heures du matin. Le prix du billet est de dix francs suisses pour la totalité de l'événement. Gratuit pour les moins de 16 ans, ce billet fait également office de titre de transport dans les transports publics pendant toute la durée de la manifestation.

La communication résolument attrayante, ainsi que le prix modeste de l'entrée sont autant de facteurs invitant un public le plus large possible à découvrir les musées lausannois. Ainsi, Lausanne décline, le temps de cette «nuit», une fête autour de la culture. D'heure en heure, les musées proposent des animations différentes sur tout le territoire de la ville. Une signalétique spécifique annonce les lieux culturels; un service de bus spécial et un service de prêt de vélos (en partenariat avec Lausanne Roule) incitent les citadins à se déplacer à travers la ville pour visiter le maximum de musées.

Depuis sa création en 2001, la Nuit des Musées lausannois continue de fidéliser son public. Le succès d'un événement, tel que la Nuit des Musées, se mesure habituellement par le taux de fréquentation. La réussite n'en est cependant que plus forte lorsque la manifestation s'adresse à toutes les tranches de la population. Le projet Accès-Cible a été lancé par l'Association Nuit des Musées (NDM) pour faciliter l'accès aux musées à des publics fréquentant peu ou ne fréquentant pas ces lieux (BoNSACK \& MÉAN 2009). Grâce à une collaboration réussie entre les 22 musées de Lausanne et Pully et de multiples partenaires, de nombreuses actions de sensibilisation, des adaptations simples et des animations ont été mises sur pied pour les publics visés par le projet. Le projet Accès-Cible avait pour objectif de faire de l'événement de la Nuit des Musées une sorte de laboratoire d'expérimentation de l'accès à la culture. Le présent article présente le projet, du point de vue de l'accessibilité pour les personnes handicapées.

\section{Une Nuit des Musées qui vise un public diversifié}

De 2000 à 2010, la fréquentation de la Nuit des Musées est passée de 4500 à plus de 16000 visiteurs. En 2004, l'événement était essentiellement fréquenté par un public ouvert à la culture et habitué des musées. L'association NDM s'est alors interrogée sur le sens d'une manifestation dont le but est de faciliter l'accès à la culture au plus grand nombre. La fréquentation est optimale, mais comment atteindre des publics absents des musées? L'événement, ponctuel, ludique et festif permet de développer et d'expérimenter des pistes de réflexion afin de démocratiser l'accès aux lieux de culture.

Trois publics ont été identifiés: les minorités étrangères, particulièrement les familles fraîchement arrivées à Lausanne; les apprentis et apprenties; les personnes ayant une déficience, que celle-ci soit intellectuelle, visuelle, auditive ou motrice.

En tant qu'événement éphémère, la Nuit des Musées permet des audaces tant dans la communication que dans les animations. Elle offre un terrain idéal pour proposer et innover autour de rencontres entre les publics ciblés et les musées. Les partenaires (p.ex. institutions muséales, représentants des associations du handicap) pouvaient s'engager sur un très court terme, sans prendre trop de risques et sans coûts financiers importants. Mais dans notre réflexion autour d'AccèsCible, la nécessité de laisser des traces de ces actions éphémères a immédiatement été posée en corollaire du projet. Il paraissait nécessaire de tisser des liens entre les publics concernés et les lieux de culture, proposer des actions susceptibles de se pérenniser et ins- 
taurer des échanges et des collaborations au-delà de l'événement. Accès-Cible agirait comme une sorte de levier ou de détonateur entre des milieux qui avaient tout à gagner à coopérer.

\section{Contexte et enjeux de l'accessibilité à la culture pour les personnes handicapées}

La question de l'intégration de la personne handicapée dans la société est indissociable de celle du changement de paradigme survenu ces dernières années, tant au niveau national qu'international, dans la manière de concevoir le handicap. Jusque dans les années 1960, confinée à la dimension individuelle de la personne, la notion de handicap traduisait un diagnostic ou une limitation fonctionnelle de l'individu. A partir des années 1980, la prise en compte de l'environnement et de la société dans la notion de handicap va modifier la donne. La montée en puissance des questions d'autonomie et d'intégration va de pair avec le constat que l'environnement et l'organisation sociale peuvent autant faciliter qu'aggraver la situation de handicap de la personne. Les nouvelles classifications du handicap, telles la Classification internationale $\mathrm{du}$ fonctionnement, $\mathrm{du}$ handicap et de la santé (CIF) de l'ORGaNISATION MONDIALE DE LA SANTÉ (2001) ou, de manière plus pragmatique, la Classification québécoise - Processus de production du handicap (PPH) (FougEYROLLAS et al. 1998) vont fournir une base de compréhension et de lecture de ce que signifie le handicap. Le PPH introduit la notion de participation sociale, qui

«s'évalue sur un continuum allant de la pleine participation sociale à la situation de handicap totale et résulte de l'interaction entre les caractéristiques personnelles de l'individu et les facteurs environnementaux de son milieu de vie» (WEBER 2004: 11).

La participation sociale porte notamment sur des domaines comme l'habitation, le travail, les loisirs et les déplacements. Dès lors, la personne handicapée n'est plus seulement considérée comme un objet de soin; elle est aussi un acteur et un consommateur de la société.

Sur le plan législatif suisse, ce changement de paradigme est concrétisé par la Loi fédérale du 13 décembre 2002 sur l'élimination des inégalités frappant les personnes handicapées, entrée en vigueur en 2004 et connue sous l'acronyme LHand. Cette loi prévoit différentes mesures pour éliminer les obstacles rencontrés dans la participation à la vie en société. Elle met l'accent sur l'accès aux bâtiments et aux services publics et, à ce titre, inclut les bâtiments dédiés à la culture. L'impulsion donnée par le législateur est certes manifeste. Cependant l'impact réel de la loi semble difficile à cerner. Selon le BuREAU FÉDÉRAL DE L'ÉGALITÉ POUR LES PERSONNES HANDICAPÉES du Département fédéral de l'intérieur suisse (2009: 8),

«cinq ans après l'entrée en vigueur de la loi, il est encore trop tôt pour évaluer ses effets sur la participation des personnes handicapées à la vie en société. La loi prévoyant l'élimination graduelle des barrières architecturales, il n'est pas non plus possible de tabler déjà sur une amélioration générale de l'accès aux constructions et installations».

Sur le plan international, l'impact des lois volontaristes en matière d'intégration semble également malaisé à évaluer et les avancées difficiles à identifier. Ainsi, en France, d'après l'ObSERvatoire NATIONAL sUR LA FORMATION, LA RECHERCHE ET L'INNOVATION SUR LE HANDICAP (2010: 64),

«la recherche reste trop souvent cantonnée aux aides techniques individuelles et ne prend pas suffisamment en compte la dimension environnementale. (...) On ne dispose ni de cadres méthodologique de référence, ni de cadres réglementaires dans ce domaine où les innovations sont particulièrement complexes à expertiser. Leur évaluation est du coup très partielle et approximative, voire inexistante».

\section{Des pratiques exemplaires comme références}

La réflexion sur l'accès à la culture, en lien avec l'égalité des droits des personnes handicapées, occupe de plus en plus le terrain des politiques publiques. Certains pays présentent une avancée indiscutable en la matière:

«Insbesondere in Grossbritannien, den USA und Australien, aber auch in Skandinavien und Frankreich haben viele Museen für Zielgruppen mit Handicaps bereits beeindruckende Massnahmen und Projekte ungesetzt. Das spezifische Know-how und die langührigen Erfahrungen, über die diese Einrichtungen verfügen, sind in zahlreichen Publikationen und Leitfäden dokumentiert. So offeriert beispielweise das Arts Council England eine breite Fülle an Publikationen zur Barrierefreiheit, ebenso wie das Council for Museums, Archives and Libraries in London» (FöHL et al. 2007:11).

En France, la première publication, en 2007, du Guide pratique de l'accessibilité, culture et handicap, connaît un succès retentissant auprès des professionnels de la culture. Christine Albanel, Ministre de la culture et de la communication, souligne, dans la préface de la réédition de l'ouvrage en 2009

«que la notion d'accessibilité concerne l'ensemble des compétences au sein des institutions culturelles. Elle implique une mise en accessibilité du cadre bâti, une attention portée à la communication et à l'information des usagers, une adaptation concertée de l'offre proposée pour 


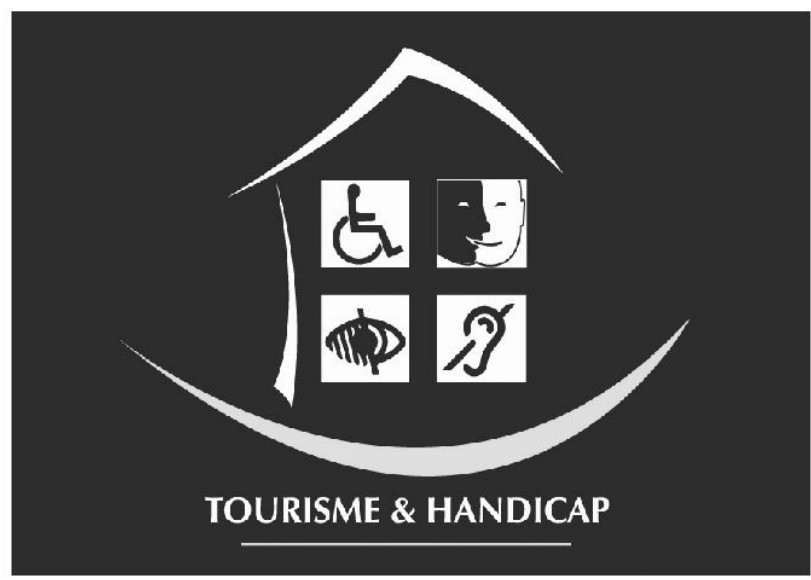

Fig. 1: Label «Tourisme et Handicap», France Label "Tourisme and Handicap», France Label «Tourismus und Handicap», Frankreich

un véritable accès aux œuvres, aux pratiques artistiques, ainsi qu'aux métiers de la culture et de la communication» (MINISTère DE LA CULTURE ET DE LA COMMUNICATION 2009:4).

Parmi les démarches entreprises, celle de la labellisation Tourisme et Handicap (fig. 1), créée par le Ministère français du tourisme, a été retenue comme référence pour le projet Accès-Cible. Cette démarche promeut la mise en accessibilité sur le plan national, par une démarche volontaire, en communiquant, par des pictogrammes spécifiques pour chacun des quatre grands groupes de personnes handicapées, les mesures qui les concernent particulièrement. L'objectif n'est pas de déterminer des situations idéales mais des prestations offrant le plus d'autonomie possible. Le label est une marque de qualité de l'accueil des personnes en situation de handicap.

Sur le plan suisse, certains lieux et actions font référence. S'agissant des lieux, citons le Musée des Transports de Lucerne, connu pour les facilités procurées aux personnes en chaise roulante, le Musée d'archéologie de Neuchâtel (Laténium) qui adapte ses visites aux personnes ayant une déficience intellectuelle ou le Musée Paul Klee de Berne qui propose des systèmes d'écoute pour les malentendants. S'agissant des actions, la distinction «Museumssterne», remise chaque année à un ou plusieurs musées de la Ville de Bâle pour récompenser une pratique innovante en matière d'accessibilité, que ce soit au niveau de l'offre culturelle ou du cadre bâti, encourage indéniablement la créativité dans ce domaine (MARIACA 2007).

Le suivi permanent de ces démarches innovantes tant sur le plan international que national a permis de disposer de points de repère pour le projet Accès-Cible et a utilement complété les travaux et les échanges entre représentants des musées et représentants des associations de personnes handicapées.

\section{Accès-Cible: comment ça marche ?}

\subsection{Les objectifs}

Accès-Cible contribue à favoriser la participation sociale des personnes handicapées en visant les objectifs suivants:

- Rendre accessible une matière, un patrimoine culturel à un type de public différent, n'ayant pas l'habitude de profiter de ce type d'offre.

- Créer des interactions entre les personnes valides et celles vivant avec un handicap. Faire découvrir au grand public une nouvelle approche, un regard différent sur le patrimoine culturel.

- Communiquer entre lieux de culture, milieux du handicap, publics. Faire exister le handicap dans le milieu des valides, solliciter de nouveaux publics.

- Apporter une valeur ajoutée pour l'ensemble du public, réfléchir à une mise en valeur différente et originale du patrimoine culturel. Une meilleure accessibilité aux lieux et au contenu est un bien pour tous.

Les postulats sur lesquels repose le projet peuvent être regroupés autour de six thèmes. Ceux-ci sont exprimés par rapport aux trois publics du projet Accès-Cible que sont les minorités culturelles, les apprentis et les personnes handicapées. En effet, le fait de ne pas maîtriser une langue ou de ne pas comprendre certains codes culurels participe, tout comme le fait d'être malvoyant ou sourd, à un «handicap» social qui rend difficile l'accès à la culture.

La participation. Le premier postulat est que toutes les actions du projet Accès-Cible s'imaginent et se concrétisent avec la participation des publics concernés. L'invitation à se rendre au musée ne se fait pas pour eux mais avec eux! La première étape du projet est donc de trouver l'interlocuteur privilégié qui agira comme relais auprès du public (Direction d'une école professionnelle, Bureau lausannois pour l'intégration des immigrés, Pro Infirmis Vaud). Sur le modèle participatif, des rencontres sont organisées entre l'équipe du musée et le public concerné, comme des personnes malvoyantes déléguées par leur association, ou des représentants de la communauté chinoise,par exemple. Ensemble, on imagine des actions en tenant compte des réalités de chacun (programmation ou disposition du musée, difficultés de se rendre dans un musée pour une personne malvoyante, réorganisation de l'entrée avec les personnes en chaise roulante), l'objectif étant 
d'instaurer un dialogue pour comprendre les désirs et limites des uns et des autres (p.ex. limites budgétaires ou en personnel pour le musée, limites architecturales pour une personne en chaise).

La mixité. Le deuxième postulat est que toutes les actions ciblées sur les publics concernés sont ouvertes à toutes et à tous. La culture participe pleinement à l'intégration sociale. Présentée au Musée de Pully (2009) et organisée avec la collaboration des minorités étrangères, l'action «Ramuz dans toutes les langues» proposait des lectures d'extraits d'ouvrages du poète vaudois Charles-Ferdinand Ramuz dans plus de onze langues différentes. Le but de cette action était de montrer la portée universelle d'un écrivain vaudois en mêlant le plaisir de découvrir RAMUz au travers de sonorités nouvelles.

La co-élaboration. Depuis 2008, Accès-Cible développe des projets avec et pour les personnes en situation de handicap. Ces collaborations sont étroitement définies avec Pro Infirmis Vaud, garant du bien-fondé des projets et interlocuteur privilégié des milieux du handicap et de la politique. C'est à ce titre que Pro Infirmis Vaud propose un cadre dans lequel les futures actions vont se développer et soutient la logistique et la communication du projet. Le relais auprès des associations de personnes handicapées est organisé de manière systématique, de la définition des mesures retenues au débriefing qui a lieu après la manifestation.

Comment le personnel de l'accueil doit-il s'adresser à une personne qui n'entend pas? Comment expliquer à une personne souffrant d'une déficience intellectuelle une maquette d'architecture? Peut-on avoir du plaisir à découvrir des pierres autrement que par la vue? Comment se déplacer dans un musée avec une chaise roulante? Et surtout comment faire se rencontrer tous les publics lors de grande affluence? Telles sont quelques-unes des questions que nous souhaitons mettre en lumière avec le projet Accès-Cible.

L'importance de l'accueil. Lors des contacts entre représentants des musées et représentants des associations de personnes handicapées, l'unanimité s'est rapidement faite autour d'un point: les actions et les aménagements mis sur pied doivent être accompagnés par une politique active d'incitation, ceci par le biais d'actions de communication et de promotion ainsi que par un accueil adéquat. Se rendre pour la première fois dans un lieu culturel sans avoir de repère implique, pour une personne ayant une déficience, une grande mobilisation de sa part. Elle ne doit donc pas être déçue ou freinée par un accueil inadéquat, tout comme une communication médiocre ne doit pas être un frein à sa démarche culturelle.
Chaque visiteur doit se sentir agréablement accueilli en arrivant dans un lieu. Selon le Ministère DE LA CULTURE ET DE LA COMMUNICATION (2009: 40-41),

«se sentir accueilli, attendu par le personnel de l'équipement culturel est une demande prioritaire des personnes handicapées, quel que soit le type de handicap. Répondre à cette attente implique que l'ensemble du personnel soit sensibilisé aux questions du handicap. Il s'agit de ne pas montrer de réticence, d'être capable d'aller vers les personnes handicapées et de s'adapter aux demandes sans être surpris ni dérouté».

Une première démarche auprès du personnel des musées a été de les sensibiliser à l'accueil. Des représentants des associations sont venus les informer sur les bonnes pratiques en matière de communication aux personnes ayant des besoins spécifiques. Des conseils simples ont été dispensés, comme la nécessité de toujours présenter son visage face à la source de lumière, de manière à faciliter la lecture labiale lorsqu'on interagit avec un malentendant, ou encore le fait d'éviter des instructions basées sur le visuel comme «les toilettes se trouvent là-bas à gauche» lorsque l'on renseigne un malvoyant.

L'invitation à se rendre aux musées. Un soin tout particulier est mis dans la communication de ces actions tant auprès du public en général (mise en évidence des actions Accès-Cible dans le programme de la Nuit des Musées, point presse et communiqué pour les médias) que dans la communication interne des publics concernés (flyers dans toutes les langues, présentation de la Nuit des Musées dans les écoles professionnelles, au Centre pédagogique pour handicapés de la vue à Lausanne, communication dans les journaux des associations des personnes ayant une déficience). Une rubrique Accès-Cible est ouverte sur le site internet de l'événement. La mention «Bienvenue aux chiens guides» est clairement indiquée par exemple. Tous les partenaires du projet sont invités au vernissage et aux diverses séances de débriefing. Un accompagnement est proposé aux associations des personnes handicapées pour soutenir leur communication interne. Un communiqué de presse ou un point presse est réalisé conjointement avec Pro Infirmis Vaud.

La sensibilisation du grand public au handicap, valeur ajoutée du projet. Toute démarche de mise en accessibilité d'un lieu ou d'un événement comporte une dimension de sensibilisation, du fait, le plus souvent, de la visibilité des mesures. Il peut s'agir de supports de signalisation ou de pictogrammes permettant de s'orienter et de prendre toute décision de cheminement, d'être informé sur des systèmes comme une boucle magnétique pour les malentendants ou des représentations partielles ou globales d'une œuvre ou 


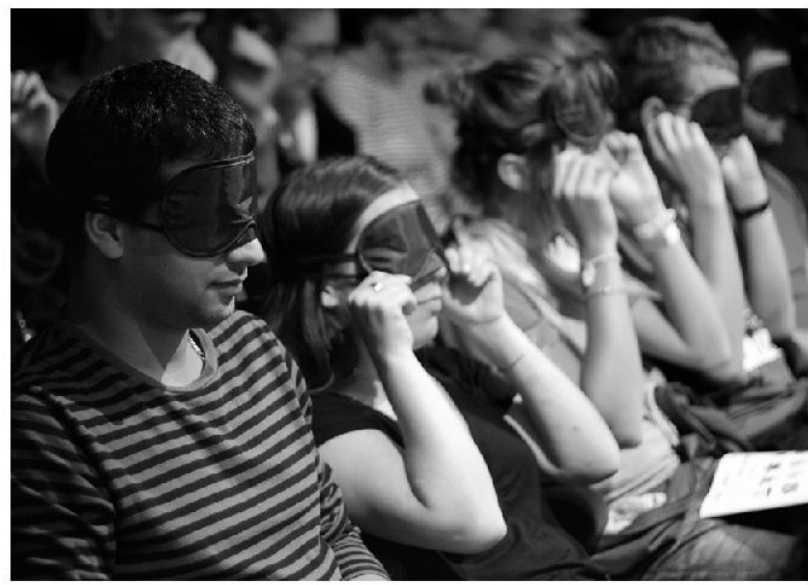

Fig. 2: «Un film dans le noir». Cinémathèque suisse, Nuit des Musées 2010

Les spectateurs sont mis en situation de non-voyants et suivent un film audio-décrit (projet Accès-Cible).

"A film in the dark». Swiss Cinemathek, Museums" Night 2010

«Ein Film im Dunklen». Schweizer Cinemathek, Nacht der Museen 2010

Photo: A. HAEnNI

d'un objet sous forme de maquette tactile. Ces mesures interpellent plus ou moins discrètement le visiteur et, à ce titre, le sensibilisent à la dimension de l'intégration. Par ces actions, Accès-Cible entend donner une visibilité à la problématique de l'accessibilité de la culture pour les personnes handicapées tout en amenant le grand public sur le terrain de nouvelles expériences. Ainsi, les Musée et jardins botaniques cantonaux proposent un Bar des signes (2008) géré par des personnes ayant une déficience auditive: le consommateur commande sa bière en langage des signes. La Cinémathèque suisse lance «Un film dans le noir» (2009 et 2010) audio-décrit pour les personnes non voyantes: tous les visiteurs sont invités à porter des lunettes opaques et découvrent le plaisir de suivre un film en privilégiant l'écoute (fig. 2). La plupart des animations invitent le visiteur à se mettre dans une situation de handicap, non sans oublier l'esprit ludique de l'événement.

En amenant le visiteur à expérimenter une situation de handicap, Accès-Cible prend le parti de quitter l'invitation discrète d'une démarche de sensibilisation pour l'amener résolument à questionner son rapport au handicap. Le projet s'inscrit dans ce que GARDou et Poizat (2007: 321) appellent de leurs vœux pour «désinsulariser le handicap»:

«Il ne convient plus de penser et d'agir en termes spécifiques pour des groupes tenus pour spécifiques. Il faut s'ap- pliquer à rendre plus confortable, à humaniser pour tous, à partir du principe universel d'accessibilité et du concept de qualité de vie».

\subsection{De la pierre odorante au coup d'reil au python: quelques exemples}

Dans un premier temps, des adaptations telles que des installations de boucles magnétiques, des réalisations de plans d'accès et de circulation, des caractères de textes grossis et des animations ont été réfléchies conjointement avec les responsables des musées et les organisations des déficiences concernées. Avec des actions spécifiques pour chaque musée, nous voulions que la personne ayant un handicap se sente accueillie et considérée comme un visiteur à part entière lors de la Nuit des Musées.

Afin de rendre directement accessible une partie du contenu de son exposition, le Musée cantonal de géologie a proposé, pour les personnes aveugles et malvoyantes mais également pour le grand public, une approche tactile et olfactive des cristaux et des minéraux (fig. 3) (2008).

Du côté du Forum d'architectures de Lausanne (f'ar), c'est un atelier de construction de maquettes qui attendait les personnes avec une déficience intellectuelle, ceci afin d'expliquer le processus de conception de manière interactive et ludique. La sensibilisation

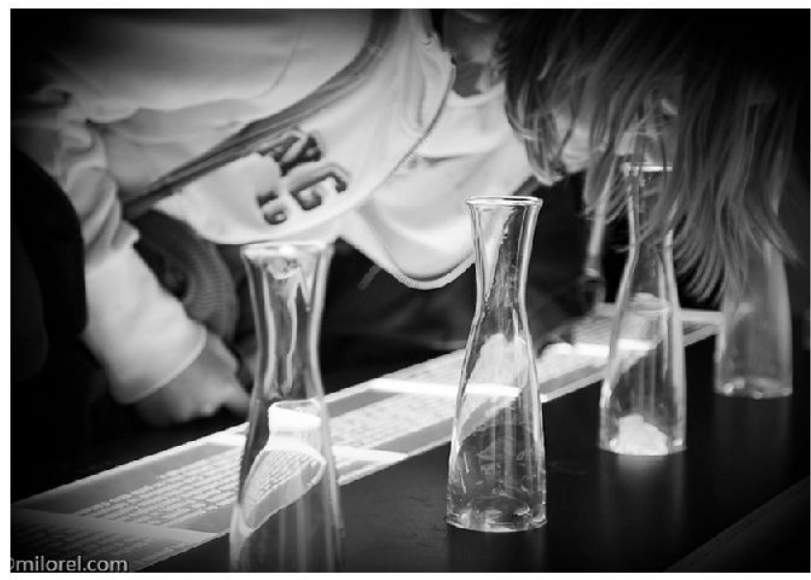

Fig. 3: «De la pierre odorante». Musée cantonal de géologie, Nuit des Musées 2008

Les visiteurs découvrent et différencient les pierres en utilisant l'odorat au lieu de la vue (projet Accès-Cible).

"About the smell of stones». Cantonal Museum of Geology, Museums' Night 2008

"Vom duftenden Stein». Kantonales Museum der Geologie, Nacht der Museen 2008

Photo: milorel.com 


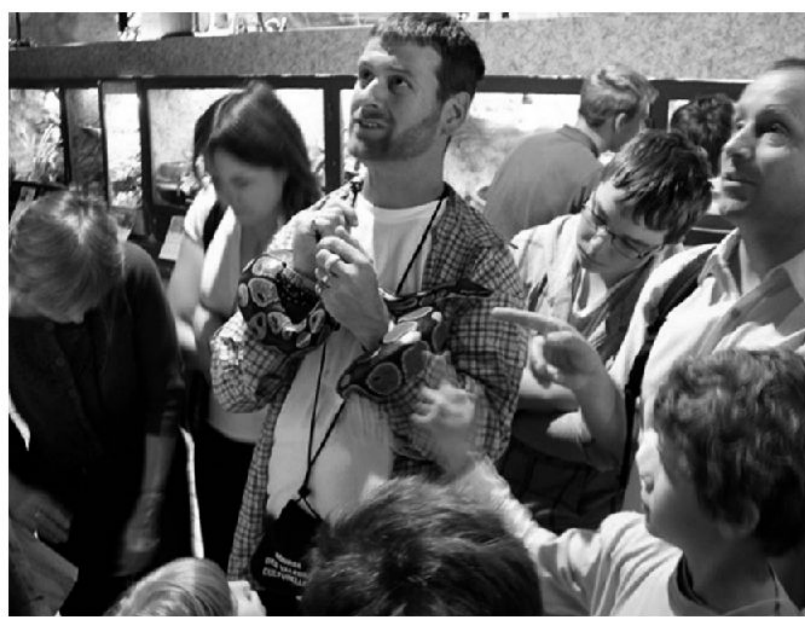

Fig. 4: «Coup d'œil au python», Vivarium de Lausanne, Nuit des Musées 2009

Un animateur ayant une déficience intellectuelle explique au public la manière de tenir un python (projet Accès-Cible).

«Eye to eye with a python». Lausanne Vivarium, Museums' Night 2009

«Aug in Aug mit der Python». Vivarium von Lausanne, Nacht der Museen 2009

Photo: L. Woringer

et l'esprit d'échange entre les divers publics ont conduit la Fédération suisse des sourds et la Fondation A Capella à collaborer avec le Musée historique de Lausanne et les Musée et jardins botaniques cantonaux: ils ont proposés des visites guidées traduites respectivement en langues des signes et en langage parlé complété $(2008,2009)$. A la Fondation Claude Verdan, les visiteurs se sont également retrouvés en situation de «handicap». Ils ont écouté dans l'obscurité totale des lectures contées par une personne aveugle (2009). Dans une optique d'échange et de partage, citons encore la contribution de personnes ayant une déficience intellectuelle tant à l'Espace des Inventions, dans la co-animation d'un atelier qu'au Vivarium dans la présentation d'un python (fig. 4) (2009). Le Musée de l'Elysée (2008), quant à lui, avait aménagé l'entrée du lieu d'exposition pour les personnes en chaise: un tapis rouge et une signalétique mettait en évidence l'entrée annexe latérale (habituellement réservée aux livreurs et aux personnes en chaise à cause de la présence d'un ascenseur). Pour l'événement, le musée a accroché des photos dans cet espace intermédiaire, le rattachant directement à la scénographie de toute l'exposition. Cet exemple mettait directement en évidence la problématique de la chaine d'accessibilité pour un public concerné et montrait comment optimiser un lieu face aux divers obstacles environnementaux.
Autant d'exemples concrets qui interrogent l'accessibilité des espaces culturels et autres lieux publics et qui confirment la direction à prendre:

«L'individu est au centre de tous les enjeux de décision politique et économique, dans la perspective d'une réponse satisfaisante, toujours optimisée, à l'ensemble de ses besoins. On peut donc facilement comprendre que l'aménagement durable sur la base des handicaps est un gage de qualité et de fréquentation optimisée des lieux. L'accessibilité est donc un élément fort de la reconquête urbaine, avec notamment le principe novateur de «l'handimension» (dimensionner l'espace et les aménagements sur la base des handicaps)» (BoDIN 1999: 8 et 2007).

\section{Un bilan très positif}

Le projet Accès-Cible montre que nous pouvons, grâce à des aménagements simples et à une meilleure connaissance des besoins et des possibilités des uns et des autres, concrétiser le vœu de personnes en situation de handicap.

Une déficience n'étant pas toujours perceptible de prime abord, il n'est pas possible d'évaluer la fréquentation des diverses éditions de la Nuit des Musées par les personnes handicapées. Nous avons toutefois observé qu'une véritable rencontre et un plaisir partagé entre les visiteurs ayant une déficience et le public en général se sont opérés lors de cette journée. Face aux diverses adaptations et aux animations, le public a été directement sensibilisé aux difficultés que rencontrent les personnes ayant une déficience dans l'approche muséale et en retire lui-même, le plus souvent, un confort accru. Ce constat confirme les observations de FöHN et al. (2007:24), qui notent que

«in den USA, Großbritannien, Frankreich und Skandinavien haben sich seit vielen Jahren zahllose Museen für Behinderte geöffnet. Sie leisten damit einen wertvollen Beitrag zur gesellschaftlichen Integration dieser Menschen und steigern gleichzeitig die Attraktivität ihrer Häuser für breite Publikumsschichten».

Certains musées partenaires ont relevé que le public en situation de handicap n'est pas venu en nombre le jour de l'événement. Ils ont cependant remarqué une fréquentation accrue de ce public dans les semaines qui ont suivi la Nuit des Musées. La foule et le bruit liés à un tel événement sont certainement des facteurs qui ont freiné les personnes handicapées à répondre à l'invitation des musées. Mais la relative faiblesse de l'échantillonnage rend difficile une généralisation et une théorisation sur l'ensemble de leur comportement.

Grâce à Accès-Cible, projet original et novateur, les personnes handicapées deviennent de véritables acteurs et 
partenaires de la Nuit des Musées. Elles ont pu directement «mettre en scène» leurs difficultés et réfléchir, avec les responsables des musées, à la manière de réaliser des actions symboliques sur l'accessibilité.

Du côté des musées partenaires, le personnel et les responsables ont montré un grand enthousiasme pour ce projet pilote. En 2007, six musées ont participé au projet. En 2010, plus de vingt musées participent à Accès-Cible. Lors de l'assemblée générale de tous les musées, une demande collective a été faite pour réaliser à leur intention un guide sur l'accueil des personnes handicapées et sur la mise en œuvre de collaborations. Leur demande a été entendue, ce guide «Boîte à outils pour promouvoir votre lieu de culture auprès des personnes en situation de handicap» est en cours de réalisation (BoNSACK \& Richoz 2011). Des contacts se prennent directement entre les musées et les représentants des associations pour les prochaines éditions et pour des actions hors Nuit des Musées. Accès-Cible a atteint son autre objectif: à partir d'un événement éphémère, laisser des traces.

\section{Bibliographie}

Bodin, F. (1999): Handicaps et sociétés, l'aménagement de l'espace comme concept d'intégration. - Thèse de géographie, UFR des Sciences de la Terre et de l'Aménagement Régional, Université de Caen Basse-Normandie.

Bodin, F. (2007): Enjeux d'une accessibilité optimisée des universités françaises SIG et Handicaps. - In: TRANSED, http://www.tc.gc.ca/fra/politique/comotred2007-pages-1106-1843.htm 11.11.2010.

Bonsack, C. \& E. MÉAN (2009): La culture accessible aux personnes ayant un handicap. - In: Museums.ch - La Revue suisse des musées 4: 104-106.

BonsaCK, C. \& M. Richoz (2011): Boîte à outils pour promouvoir votre lieu de culture auprès des personnes en situation de handicap. - Lausanne: Nuit des Musées et Pro Infirmis Vaud (à paraître).

BurEaU FÉdÉRAL DE L'ÉGALITÉ POUR LES PERSONNES HANDICAPÉES (BFEH) (2009): Egalité des personnes handicapées 2004-2009. Tendances et enjeux. - Berne: Département fédéral de l'intérieur.

Föhl, P., Erdrich, S., Hartmut, J. \& K. Mass (2007): Das barrierefreie Museum. Theorie und Praxis einer besseren Zugänglichkeit - Ein Handbuch. - Bielefeld: transcript Verlag.

Fougeyrollas, P., Cloutier, R., Bergeron, H., Côté, J. \& G. ST-Michel (1998): Classification québécoise. Processus de production du handicap. - Québec: Réseau International sur le Processus de Production du Handicap.

Gardou, C. \& D. Poizat (2007): Désinsulariser le handicap - quelles ruptures pour quelles mutations culturelles? - Ramonville Sainte-Agnès: Érès.
MARIACA, P. (2007): Introduction au projet d'accueil de publics différents à la nuit des musées 2008. - Lausanne: Pro Infirmis Vaud.

Ministère DE LA CUlture ET DE LA COMMUNiCATION (2009): Culture et Handicap. Guide pratique de l'accessibilité. - Paris: Ministère de la culture et de la communication.

OBSERVATOIRE NATIONAL SUR LA FORMATION, LA RECHERCHE ET L'INNOVATION SUR LE HANDICAP (2010): Rapport 2009. - Paris.

ORganisation mONdiAlE dE LA SANTÉ (2001) : Classification internationale du fonctionnement, du handicap et de la santé. - Genève: OMS.

WEBER, P. (2004): Travail social et handicap: de l'inclusion à la participation sociale. - In: Développement humain, handicap et changement social 13, 1-2: 1020 .

\section{Sites web}

www.lanuitdesmusees.ch

www.tourisme-handicaps.org. - Site de l'Association Tourisme et Handicaps

www.museumssterne.ch. - Site de la distinction Museumssterne

www.edi.admin.ch/ebgb. - Site du Bureau fédéral de l'égalité pour les personnes handicapées

\section{Résumé: Le projet Accès-Cible de la Nuit des Musées lausannois: une expérimentation pour promouvoir l'accès des personnes handicapées à la culture}

Chaque année, plus de 16000 personnes fréquentent la Nuit des Musées, manifestation lausannoise connue pour son esprit décalé et la forte proportion de jeunes parmi ses visiteurs. Avec le projet Accès-Cible, l'Association de la Nuit des Musées, en collaboration avec Pro Infirmis Vaud, utilise ce formidable événement culturel et social comme déclencheur d'idées et d'expériences autour de l'accessibilité de l'évènement aux personnes handicapées. Le projet Accès-Cible vise un double objectif: permettre l'accessibilité aux personnes handicapées et sensibiliser le grand public à l'intégration et à la participation sociale des personnes ayant une déficience. Les diverses actions et mesures sont conçues et réalisées en partenariat étroit avec les personnes en situation de handicap. Elles sont également communiquées conjointement au grand public via les médias et aux associations d'entraide de personnes handicapées via leur communication interne. Largement inspirées des pratiques novatrices des musées français et du label national français «Tourisme et Handicap», les mesures concernent autant les personnes à mobilité réduite que les personnes ayant une déficience visuelle, auditive ou intellectuelle. L'importance de la sensibilisation du personnel de l'accueil a été mise en évidence pour l'ensemble de ces groupes. Ce projet, d'abord expérimental, a ensuite été affiné, 
pour déboucher sur de nouvelles pratiques qui ont valeur d'exemples, notamment la rencontre entre le public ayant une déficience et le public valide autour d'une œuvre ou d'une matière dont la présentation est adaptée aux besoins des personnes handicapées. Les éditions 2008,2009 et 2010 ont connu un grand succès et les 22 musées lausannois se montrent de plus en plus ouverts à collaborer sur d'autres projets.

Mots-clés: accessibilité, handicap, culture, musées, public, Lausanne

\begin{abstract}
The «ACCES-CIBLE» project: opening doors to cultural events for people with disabilities Since 2008 , people with different disabilities have been encouraged to participate in Lausanne's «Museums' Night», an annual off-beat event which attracts about $20^{\prime} 000$ people. With «ACCES-CIBLE», Lausanne's Museums' Night committee, in collaboration with Pro Infirmis Vaud, hope to take advantage of this exceptional cultural and social event to trigger ideas and experimentation around accessibility. The project «ACCES-CIBLE» has a dual purpose: it aims to increase accessibility of the event to people with disabilities and raise the awareness of the general public of need and means to integrate people with disabilities and encourage their social inclusion. The various activities and measures are planned and implemented in close partnership with people living with disabilities. Inspiration for the project was drawn from the innovative practices of French museums and the French national label «Tourisme \& handicap», which targets people with different disabilities, be these visual, auditory, physical or cognitive. Particular emphasis has been placed in this project on the training of staff at participating locations. This project, which started as an experiment, has developed and grown, leading to the initiation of innovative projects, such as the informal meeting of people with and without disabilities during the Museums' Night around a piece of work or a presentation adapted to both groups.
\end{abstract}

Keywords: accessibility, disabilities, culture, museums, public, Lausanne

\section{Zusammenfassung: Das Projekt ACCES-CIBLE - neue Zugangsmöglichkeiten für Behinderte zur Kultur}

Seit 2008 wird die Zugänglichkeit der Lausanner «Langen Nacht der Museen» für behinderte Menschen ständig verbessert. Über 16'000 Personen besuchen jedes Jahr die etwas schräge und besonders bei jungen Menschen beliebte Veranstaltung. Die Vereinigung «Lange Nacht der Museen» hat in Zusammenarbeit mit Pro Infirmis Waadt das Projekt «Accès-Cible» lanciert: Das grossartige kulturelle und gesellschaftliche Event wird für Ideen und Experimente rund um die Zugänglichkeit genutzt. Das Projekt «AccèsCible» verfolgt zwei Ziele: Es will einerseits Museen für behinderte Menschen zugänglich machen und andererseits die Öffentlichkeit für die Integration und gesellschaftliche Teilhabe dieser Bevölkerungsgruppe sensibilisieren. Die verschiedenen Aktionen und Massnahmen werden in enger Zusammenarbeit mit den behinderten Menschen erarbeitet und umgesetzt. Gleichzeitig werden die Öffentlichkeit über die Medien und die Menschen mit Behinderung über ihre Selbsthilfeorganisationen informiert. Bei ihren Aktionen haben sich die Projektverantwortlichen weitgehend auf die innovative Arbeit der französischen Museen und auf das französische Label «Tourisme et Handicap» gestützt. Die Massnahmen richten sich nicht nur an Gehbehinderte, sondern auch an Seh- und Hörgeschädigte sowie an geistig behinderte Menschen. Auf die Sensibilisierung des Empfangspersonals für die Bedürfnisse dieser verschiedenen Zielgruppen wurde besonders grosser Wert gelegt. Nach der Versuchsphase wurde das Projekt verfeinert und neue, beispielhafte Lösungen entstanden. So konnten sich behinderte und nichtbehinderte Menschen beim Betrachten von Kunstwerken und anderen Ausstellungsstücken auf gleicher Augenhöhe begegnen.

Schlüsselwörter: Zugänglichkeit, Handicap, Kultur, Museen, Öffentlichkeit, Lausanne

Carine Bonsack, Plates-Bandes, Valentin 34 et demi, CH-1004 Lausanne, Suisse.

Monique Richoz, Pro Infirmis Vaud, Rue du GrandPont 2bis, Case postale 7137, CH-1002 Lausanne, Suisse.

e-mail:

carine.bonsack@plates-bandes.ch

monique.richoz@proinfirmis.ch
Manuskripteingang/received/manuscrit reçu le 30.7.2010
Annahme zum Druck/accepted for publication/accepté pour publication: 15.12 .2010 